Curr Opin Chem Biol. 2012 August ; 16(3-4): 253-259. doi:10.1016/j.cbpa.2012.05.179.

\title{
Synthetic in vitro circuits
}

\author{
Adam J. Hockenberry ${ }^{1,2}$ and Michael C. Jewett ${ }^{1,2,3,4, *}$ \\ ${ }^{1}$ Interdepartmental Biological Sciences Graduate Program, Northwestern University, 2205 Tech \\ Drive, Evanston, IL 60208, USA \\ ${ }^{2}$ Chemistry of Life Processes Institute, Northwestern University, 2170 Campus Drive, Evanston, \\ IL 60208, USA \\ ${ }^{3}$ Department of Chemical and Biological Engineering, Northwestern University, 2145 Sheridan \\ Road, Evanston, IL 60208, USA \\ ${ }^{4}$ Member, Robert H. Lurie Comprehensive Cancer Center, Northwestern University, Feinberg \\ School of Medicine, Northwestern University, 303 E. Superior, Chicago, IL 60611, USA
}

\begin{abstract}
Inspired by advances in the ability to construct programmable circuits in living organisms, in vitro circuits are emerging as a viable platform for designing, understanding, and exploiting dynamic biochemical circuitry. In vitro systems allow researchers to directly access and manipulate biomolecular parts without the unwieldy complexity and intertwined dependencies that often exist in vivo. Experimental and computational foundations in DNA, DNA/RNA, and DNA/RNA/ protein based circuitry have given rise to systems with more than 100 programmed molecular constituents. Functionally, they have diverse capabilities including: complex mathematical calculations, associative memory tasks, and sensing of small molecules. Progress in this field is showing that cell-free synthetic biology is a versatile training ground for understanding native biological circuits and engineering novel functionality.
\end{abstract}

\section{Introduction}

Breakthroughs in our ability to read and write DNA have enabled researchers to construct sophisticated, circuit-like behavior in living systems [1]. More than ten years ago, pioneering efforts by Elowitz and Leibler and Gardner et al. [2,3] provided a conceptual framework for viewing cells as a system of interacting circuits - structures composed of several elements that are capable of information processing by accepting an input, executing a series of logical computations, and producing an output. This framework, championed by many synthetic biologists [4], has inspired new applications and shed light on our understanding of fundamental biological phenomena. Nevertheless, in vivo circuitry is often unpredictable. Even if the functions of individual 'parts' are known, they may not work as expected in different cellular contexts due to intertwined circuit dependencies and crosstalk between biomolecules. Thus, the complexity of successfully implemented in vivo circuits to date is limited in size and scope to tens of molecules [4]. This pales vastly in comparison to

\footnotetext{
(C) 2012 Elsevier Ltd. All rights reserved

*m-jewett@northwestern.edu.
}

Publisher's Disclaimer: This is a PDF file of an unedited manuscript that has been accepted for publication. As a service to our customers we are providing this early version of the manuscript. The manuscript will undergo copyediting, typesetting, and review of the resulting proof before it is published in its final citable form. Please note that during the production process errors may be discovered which could affect the content, and all legal disclaimers that apply to the journal pertain. 
the simplest living organisms, which consist of several hundred to thousands of genes, noncoding RNA species, and small molecules.

As a complement to in vivo-based approaches, in vitro systems provide an unprecedented freedom to modify and control biochemical systems for technological applications and to understand the design principles of biological circuits. Removing genetic regulation, for instance, drastically reduces complexity by negating a cells' ability to adapt and evolve [5]. For decades, in vitro systems have made integral contributions to biological research in both discovery and technology - from discovery of the genetic code [6] to the development of core techniques such as the Polymerase Chain Reaction [7] as well as disruptive technologies for manufacturing-scale cell-free protein synthesis [8]. Now, the study of complex biological circuits is emerging as a novel application of in vitro systems that can complement and guide in vivo studies.

This review will focus on recent developments in the field of synthetic in vitro circuits over the last 3 years. We first examine minimal nucleic-acid based systems that offer unparalleled experimental flexibility and predictability. Next, we discuss circuits created in hybrid systems - containing purified enzymes and nucleic acids. We end our discussion of in vitro circuits on complex systems that are capable of transcription and translation.

\section{Nucleic acid systems}

While the classical sense of the word "circuit" conjures up images electricity flowing through transistors, DNA is capable of both carrying information and performing computations on that information $[9,10]$. Circuits constructed in this manner have relatively few possible interactions and points of control (Figure 1 (top)) making their quantitative description more manageable and comprehensive. They provide theoretical insight into the generalized function of chemical circuits based on diffusion and stochastic binding principles that must be understood to make genetic circuit design a predictive science.

Dramatic increases in circuit complexity in DNA systems over the last three years partly stem from the development of 'seesaw' gates that make use of toehold exchange reactions (Figure 2A,B) [11-13••]. In classical toehold displacement reactions, a single-stranded DNA (ssDNA) molecule displaces one strand of a double-stranded DNA (dsDNA) complex by binding to a complementary short overhang (toehold) region on the dsDNA. The ssDNA (input) displaces the previously bound strand of DNA (output), which can then serve as an incoming signal for another reaction. From this starting point, researchers have built a diversity of nano-scale devices and circuits capable of complex computation (see [14•] and [15] for more comprehensive reviews).

In the case of toehold exchange reactions, the reverse reaction is made possible by including a toehold design on both sides of the dsDNA molecule (Figure 2A). Only two cascaded seesaw gates are required to produce the OR and AND logical functions (Figure 2B) and more complex cascades can compute NOT, NAND, NOR, and XOR. To show the scalability of these gate units, Qian et al. constructed a neural network consisting of 112 DNA strands that demonstrates associative memory capable of answering 81 possible questions [16], as well as a 130-stranded DNA circuit capable of computing the square root of a 4 digit binary number [17•].

Models derived from experimental investigations $[11,12]$ are increasingly accurate at predicting the kinetics of strand displacement reactions and have allowed construction of increasingly complex implementations. Further, experiments guided by thermodynamic models have verified designs that perform within a $20^{\circ} \mathrm{C}$ range of temperatures, $1-5 \mu \mathrm{M}$ concentrations of nucleic acid, and $1-47 \mathrm{mM} \mathrm{Mg}^{2+}[18 \cdot]$. This robustness illustrates that the 
understanding gained from these systems may be generalized to a range of conditions and not limited to particular experimental set-ups. A suite of computational tools, theoretical frameworks and even dedicated programming languages can be used to design and analyze DNA circuits [19-23]. Concurrently, novel theoretical possibilities [24] and practical implementations are continually being developed such as those utilizing hairpins [25,26], cooperative binding [27], deoxyribozymes [28,29], photochemical activation [30] and associative toehold activation [31].

Importantly, studying strand displacement circuits in vitro has led to practical uses that would have been difficult to anticipate prior to the expansion of this largely theoretically motivated work. Researchers have shown that in vitro DNA circuits can amplify signals and detect RNA and small molecule analytes using different reporting methods [32]. Further, an area of research that is likely to expand involves using DNA circuits as visualization tools for imaging DNA based reaction cascades [33], mRNA's [34] and reversibly imaging proteins [35].

\section{Hybrid systems}

Understanding and exploiting transcriptional logic is a complex problem that unites many fields of biology, but the vast number of interacting species and circuit cross talk within cells makes it difficult to characterize individual transcriptional gates. In vitro hybrid systems composed of DNA templates and purified enzymes, however, allow researchers to isolate the performance of small circuits before exploring how they interact within larger biochemical systems [36-39]. DNA still acts as the logic gate for signal integration (see Figure $3 \mathrm{~A}$ for sample AND gate implementation), but the inputs and outputs can be DNA, RNA, or metabolites.

Hybrid systems are able to amplify signals, produce new signals, and degrade waste products - functionality that is difficult to implement in nucleic acid systems. Pioneering studies in 2011 highlight the diversity of these circuits, and the fact that similar high-level designs can be implemented in different ways. Montagne et al. created a system (the "Oligator") capable of sustained oscillations using DNA polymerase, a nicking enzyme, and an exonuclease that were responsible for amplification, propagation, and destruction of information carrying DNA oligonucleotides [40••]. This circuitry used only three template strands and three enzymes but was able to couple a positive feedback and a delayed negative feedback loop in a computationally predictable manner that used readily attainable parameters.

Using a different molecular implementation, Kim and Winfree produced a system capable of sustained oscillations by using RNA polymerase and RNaseH to produce and degrade cascading DNA/RNA signals [41 • $]$. They showed oscillations using three distinct designs: a seven-stranded two-switch negative feedback oscillator, an amplified negative feedback oscillator which included the use of a positive feedback system, and finally a novel molecular implementation of a three-switch ring oscillator that was the focus of pioneering in vivo studies [2]. Franco et al. later expanded on this oscillating system and displayed potential applications by using the negative feedback oscillator as a timing device to separately activate DNA nanotweezers and produce an RNA aptamer [42••]. As with Montagne et al., modeling was integral to understanding system behavior and parameters including concentrations and sequences - that would produce desired results. Merely by introducing the novel interaction that was required to time their device, the oscillations were severely restricted compared to the original system. This problem of coupling distinct systems was elegantly overcome through the use of an insulator circuit that isolated the output signal and prevented it from disrupting the timing device. 
While the previous studies showed successful implementations of designs, they varied in their predictability and quantitative understanding of the systems. Deviations from kinetic models, especially with regards to the repressilator implementation, highlight the shortcoming of our knowledge and make a strong case for studying these systems before attempting to explain more complex cellular oscillators. For instance, incomplete degradation products may partially inhibit reactions in ways that are difficult to predict. In addition, small differences in transcription and degradation rates can compound over time and result in unstable oscillations with growing amplitudes or mono-stable behavior. Nevertheless, studies aimed at predicting and modeling more complex systems show a clear path forward for creating autoregulatory switches [43], neural networks [44], incoherent feed forward loops [45], pattern formation reactions [46•], as well as transcriptional rate regulation based on self-repression [47] and positive feedback [48].

As with DNA circuits, hybrid circuits have a variety of potential applications that are just beginning to emerge. In work that stems partly from initial theoretical and in vitro research[49], hybrid circuits were demonstrated inside Drosophila embryo lysates [50] providing insight on the functioning of circuits in a much more complex chemical environment. Several studies showed successful in vivo implementations of logical computing for potential applications in diagnosis and therapeutics [51,52].

\section{Complete systems}

Although previously mentioned studies may include the use of proteins, complete (either purified, or extract based) systems produce proteins in vitro and integrate them into circuits. Logical integration occurs at the DNA level but inputs and outputs may be DNA, RNA, protein, or metabolites - all of which may be produced, degraded, or modified in response to signals. Importantly, the diversity of interactions means that there are many more steps that must be understood for predictive implementation of complex circuits (Figure 1). However, these extra steps are also novel points of control that, once thoroughly characterized, may be exploited by researchers to construct highly complex logic systems.

Complete cell-free circuits began with the seminal work in 2003 of Noireaux et al. who showed that activating and inhibitory proteins could be produced in vitro and that one-, two-, and three-stage cascades could be created using these proteins as circuit elements [53]. Rather than relying on orthogonal promoters from these previous studies, Shin and Noireaux have recently recreated [54] and comprehensively characterized the endogenous sigma factor-based regulation system for use in Escherichia coli extracts [55••]. This allowed for the construction of the logical AND gate (Figure 3B), negative feedback loops, and multiple stage cascades. They also demonstrated the modularity of their toolkit for future applications of in vitro metabolic engineering and as a possible testing ground for in vivo recombinant circuit design. In complementary work, Asahara and Chong expressed promoter subunits and sigma factors in a single tube and showed promoter specific transcription initiation from solely in vitro translated and assembled products [56]. Taking an alternative route towards a similar goal, Karig et al. expanded on the original work of Noireaux et al. by showing that tool-kits based on orthogonal T7 promoters are capable of complex computations by characterizing several inducible and repressible variants and creating a negative feedback loop in vitro [57].

An overarching theme of these studies is the precise characterization of individual circuit elements, which are then implemented into complex cascades where their interactions may be controlled. While the search for biological 'parts' has proven fruitful for in vivo synthetic biologists, many of these parts are still highly context dependent. In cell-free systems, these parts exist in a context outside of cellular adaptation and evolution and the results are 
therefore expected to be more tunable and reproducible. However, before controlling protein levels based on logic integration, we must understand the range of possible points of control and parameters that are important to steady-state protein levels. Karig et al. looked at the effects of ribosome binding sites and plasmid usage on target protein levels that were essential components of their circuit [57]. Though they did not explicitly recreate circuits, in separate studies Ahn et al. used cell-free systems to characterize the effects of 3' transcript stability, fusion partner leader sequences, and initial codons on protein production rate and steady state levels [58-60]. Many of these studies look at determinants of single protein production, but Du et al. have taken this work one step farther and studied the complications that arise when trying to express multiple proteins in vitro [61]. Karzbrun et al. took a step towards integrating the effects of these different components by creating a computational framework for predicting protein levels [62•]. With this knowledge, researchers may be able to implement logical control at specific processes to enable diverse circuit designs and some even dream of minimal cells [63].

Finally, in what we believe will be an increasing trend during the coming years, several studies have designed and analyzed circuits in vitro and successfully implemented those designs in vivo. Saito et al. developed a translational regulatory system based on L7Ae, an archael ribosomal protein, binding to mRNA molecules [64*0]. They describe different mRNAs that are capable of activation and repression in response to the same protein input. This elegant study highlights the diversity of possible inputs that can be accepted by circuits composed in complete systems and exploits a unique control point that is only available in systems capable of translation. Importantly, their design was first tested in a purified cellfree translational system before being successfully implemented inside of a human cell-line. Similarly, Karig et al. tested their T7-driven negative feedback constructs in a purified cellfree translation system, cell extracts, and E. coli cells and showed the expected functionality across all systems [57]. These studies indicate that in addition to an increasing number of in vitro applications $[65,66]$, in vitro environments can be a training ground for in vivo circuit design.

\section{Conclusion}

There has been dramatic growth in the construction, complexity, and predictability of cellfree biological circuits over the past three years. This growth underscores the importance of cell-free systems as a novel environment for engineering and analyzing complex biological circuits at the molecular level. In addition, designs that rely on DNA strand displacement reactions are yielding tremendous insight into the emergent behavior of complex chemical systems that lies at the heart of cellular life. Recent growth in this field also reflects an increasing interest in exploiting the unprecedented freedom of design afforded by cell-free biology for compelling applications [5,65-67]. The in vitro circuits described above, being structurally and functionally diverse, are poised to impact chemical, physical, biological, and computational research in diverse and unpredictable ways. This is precisely what makes their continued development necessary and exciting.

\section{Acknowledgments}

We are grateful to Erik Winfree for advice and comments. Special thanks also to C. E. Hodgman, S. Kamper, A. Pah, K. Tanaka, L. Timmerman, J. Wang and P. Winter for thoughtful comments on the manuscript. We gratefully acknowledge funding from the National Institutes of Health (Grant Number R00GM081450), the National Academies Keck Futures Initiative (Grant Number NAFKI-SB5), the National Science Foundation (Grant Number MCB-0943393), the Office of Naval Research (Grant Number N00014-11-1-0363), the DARPA YFA Program (Grant Number N66001-11-1-4137), the Army Research Office (Grant Number W911NF-11-1-044), and the NSF Materials Network Grant (Grant Number 1108350). 


\section{References}

- of special interest

•• of outstanding interest

1. Khalil AS, Collins JJ. Synthetic biology: applications come of age. Nature Reviews Genetics. 2010; 11:367-79.

2. Elowitz MB, Leibler S. A synthetic oscillatory network of transcriptional regulators. Nature. 2000; 403:335-8. [PubMed: 10659856]

3. Gardner TS, Cantor CR, Collins JJ. Construction of a genetic toggle switch in Escherichia coli. Nature. 2000; 403:339-342. [PubMed: 10659857]

4. Purnick PEM, Weiss R. The second wave of synthetic biology: from modules to systems. Nature Reviews Molecular Cell Biology. 2009; 10:410-22.

5. Hodgman CE, Jewett MC. Cell-free synthetic biology $\square$ : Thinking outside the cell. Metabolic Engineering. 2011 doi:10.1016/j.ymben.2011.09.002.

6. Nirenberg MW, Matthaei JH. The Dependence of Cell-Free Protein Synthesis in E. Coli Upon Naturally Occurring or Synthetic Polyribonucleotides. Proceedings of the National Academy of Sciences of the United States of America. 1961; 47:1588-1602. [PubMed: 14479932]

7. Saiki RK, Scharf S, Faloona F, Mullis KB, Horn GT, Erlich HA, Arnheim N. Enzymatic amplification of -globin Genomic Sequences and Restriction Site Analysis for Diagnosis of Sickle Cell Anemia. Science (New York, N.Y.). 1985; 230:1350-1354.

8. Zawada JF, Yin G, Steiner AR, Yang J, Naresh A, Roy SM, Gold DS, Heinsohn HG, Murray CJ. Microscale to manufacturing scale-up of cell-free cytokine production--a new approach for shortening protein production development timelines. Biotechnology and bioengineering. 2011; 108:1570-8. [PubMed: 21337337]

9. Adleman LM. Molecular Computation of Solutions to Combinatorial Problems. Science (New York, N.Y.). 1994; 266:1021-1024.

10. Seelig G, Soloveichik D, Zhang DY, Winfree E. Enzyme-free nucleic acid logic circuits. Science (New York, N.Y.). 2006; 314:1585-8.

11. Zhang DY, Winfree E. Control of DNA strand displacement kinetics using toehold exchange. Journal of the American Chemical Society. 2009; 131:17303-14. [PubMed: 19894722]

12. Zhang DY, Winfree E. Robustness and modularity properties of a non-covalent DNA catalytic reaction. Nucleic acids research. 2010; 38:4182-4197. [PubMed: 20194118]

13 •. Qian L, Winfree E. A simple DNA gate motif for synthesizing large-scale circuits. Journal of the Royal Society, Interface / the Royal Society. 2011; 8:1281-1297. This work introduced seesaw gates as a potential architecture for synthesis of large-scale circuits and includes the theoretical foundations for how these circuits function.

14 . Zhang DY, Seelig G. Dynamic DNA nanotechnology using strand-displacement reactions. Nature chemistry. 2011; 3:103-13. This comprehensive review article focuses entirely on DNA reactions, their application to circuits, as well as the creation of nano-scale structure and machines.

15. Chen X, Ellington AD. Shaping up nucleic acid computation. Current opinion in biotechnology. 2010; 21:392-400. [PubMed: 20538451]

16. Qian L, Winfree E, Bruck J. Neural network computation with DNA strand displacement cascades. Nature. 2011; 475:368-372. [PubMed: 21776082]

17•. Qian L, Winfree E. Scaling Up Digital Circuit Computation with DNA Strand Displacement Cascades. Science. 2011; 332:1196-1201. [PubMed: 21636773] In this article, the authors describe the first large-scale experimental implementation of seesaw gate-based circuits. This work culminates in a circuit capable of computing the square root of a four digit binary number.

18 . Zhang DY, Chen SX, Yin P. Optimizing the specificity of nucleic acid hybridization. Nature chemistry. 2012; 4:208-214.The authors analyze the thermodynamic properties of nucleic acid hybridization reactions over a range of experimental conditions to develop highly sensitive and robust probes for potential applications in strand displacement reactions. 
19. Zadeh JN, Steenberg CD, Bois JS, Wolfe BR, Pierce MB, Khan AR, Dirks RM, Pierce NA. NUPACK $\square$ : Analysis and Design of Nucleic Acid Systems. Journal of Computational Chemistry. 2010; 32:170-173. [PubMed: 20645303]

20. Phillips A, Cardelli L. A programming language for composable DNA circuits. Journal of the Royal Society, Interface / the Royal Society. 2009; 6:S419-36.

21. Lakin MR, Parker D, Cardelli L, Kwiatkowska M, Phillips A. Design and analysis of DNA strand displacement devices using probabilistic model checking. Journal of the Royal Society, Interface / the Royal Society. 2012 doi:10.1098/rsif.2011.0800.

22. Lakin MR, Youssef S, Cardelli L, Phillips A. Virtual DSD: a design and analysis tool for DNA strand displacement systems. Bioinformatics. 2011; 27:3211-3213. [PubMed: 21984756]

23. Cardelli L. Strand algebras for DNA computing. Natural Computing. 2010; 10:407-428.

24. Soloveichik D, Seelig G, Winfree E. DNA as a universal substrate for chemical kinetics. Proceedings of the National Academy of Sciences of the United States of America. 2010; 107:5393-8. [PubMed: 20203007]

25. Yin P, Choi HMT, Calvert CR, Pierce NA. Programming biomolecular self-assembly pathways. Nature. 2008; 451:318-22. [PubMed: 18202654]

26. Genot AJ, Bath J, Turberfield AJ. Reversible logic circuits made of DNA. Journal of the American Chemical Society. 2011; 133:20080-3. [PubMed: 22111514]

27. Zhang DY. Cooperative hybridization of oligonucleotides. Journal of the American Chemical Society. 2011; 133:1077-86. [PubMed: 21166410]

28. Macdonald J, Li Y, Sutovic M, Lederman H, Pendri K, Lu W, Andrews BL, Stefanovic D, Stojanovic MN. Medium scale integration of molecular logic gates in an automaton. Nano letters. 2006; 6:2598-603. [PubMed: 17090098]

29. Eckhoff G, Codrea V, Ellington AD, Chen X. Beyond allostery $\square$ : Catalytic regulation of a deoxyribozyme through an entropy-driven DNA amplifier. Journal of Systems Chemistry. 2010; $1: 1-6$.

30. Prokup A, Hemphill J, Deiters A. DNA computation: a photochemically controlled and gate. Journal of the American Chemical Society. 2012; 134:3810-5. [PubMed: 22239155]

31. Chen X. Expanding the rule set of DNA circuitry with associative toehold activation. Journal of the American Chemical Society. 2012; 134:263-71. [PubMed: 22129141]

32. Li B, Ellington AD, Chen X. Rational, modular adaptation of enzyme-free DNA circuits to multiple detection methods. Nucleic acids research. 2011 doi:10.1093/nar/gkr504.

33. Jungmann R, Steinhauer C, Scheible M, Kuzyk A, Tinnefeld P, Simmel FC. Single-molecule kinetics and super-resolution microscopy by fluorescence imaging of transient binding on DNA origami. Nano letters. 2010; 10:4756-61. [PubMed: 20957983]

34. Choi HMT, Chang JY, Trinh LA, Padilla JE, Fraser SE, Pierce NA. Programmable in situ amplification for multiplexed imaging of mRNA expression. Nature Biotechnology. 2010; 28:1208-1212.

35. Duose DY, Schweller RM, Zimak J, Rogers AR, Hittelman WN, Diehl MR. Configuring robust DNA strand displacement reactions for in situ molecular analyses. Nucleic acids research. 2011 doi:10.1093/nar/gkr1209.

36. Wlotzka B, McCaskill JS. A molecular predator and its prey: coupled isothermal amplification of nucleic acids. Chemistry \& biology. 1997; 4:25-33. [PubMed: 9070425]

37. Dittmer WU, Simmel FC. Transcriptional Control of DNA-Based Nanomachines. Nano Letters. 2004; 4:689-691.

38. Dittmer WU, Kempter S, Rädler JO, Simmel FC. Using gene regulation to program DNA-based molecular devices. Small (Weinheim an der Bergstrasse, Germany). 2005; 1:709-12.

39. Kim J, White KS, Winfree E. Construction of an in vitro bistable circuit from synthetic transcriptional switches. Molecular systems biology. 2006; 2:68. [PubMed: 17170763]

40••. Montagne K, Plasson R, Sakai Y, Fujii T, Rondelez Y. Programming an in vitro DNA oscillator using a molecular networking strategy. Molecular Systems Biology. 2011; 7:1-7.This work demonstrates the implementation of a remarkably simple and predictable oscillator using DNA biochemistry and purified enzymes. 
41••. Kim J, Winfree E. Synthetic in vitro transcriptional oscillators. Molecular Systems Biology. 2011; 7:1-15.In this work, the authors experimentally implement and computationally analyze three separate transcriptional oscillator designs.

42••. Franco E, Friedrichs E, Kim J, Jungmann R, Murray R, Winfree E, Simmel FC. Timing molecular motion and production with a synthetic transcriptional clock. Proceedings of the National Academy of Sciences of the United States of America. 2011; 108This paper utilized a transcriptional oscillator to show successful coupling of two biochemical systems via signal isolation of the downstream processes.

43. Subsoontorn P, Kim J, Winfree E, Systems N. Bistability of an In Vitro Synthetic Autoregulatory Switch. arXiv: 1101.0723v1. 2011

44. Kim J, Hopfield JJ, Winfree E. Neural network computation by in vitro transcriptional circuits. Advances in Neural Information Processing Systems. 2004; 17:681-688.

45. Kim J, Murray RM. Analysis and design of a synthetic transcriptional network for exact adaptation. IEEE Biomedical Circuits and Systems Conference. 2011:345-348.

46•. Simpson ZB, Tsai TL, Nguyen N, Chen X, Ellington AD. Modelling amorphous computations with transcription networks. Journal of the Royal Society, Interface / the Royal Society. 2009; 6:S523-S533.The authors provide a theoretical framework for viewing transcriptional networks as biochemical computers similar to semi-conductors and suggest advantages and possible routes forward for the field.

47. Franco, E.; Forsberg, P-ola; Murray, RM. Design, modeling and synthesis of an in vitro transcription rate regulatory circuit. American Control Conference; 2008. p. 2786-2791.

48. Franco, E.; Murray, RM. Design and performance of in vitro transcription rate regulatory circuits. 47th IEEE Conference on Decision and Control; Ieee; 2008. p. 161-166.

49. Benenson Y, Paz-Elizur T, Adar R, Keinan E, Livneh Z, Shapiro E. Programmable and autonomous computing machine made of biomolecules. Nature. 2001; 414:430-4. [PubMed: 11719800]

50. Xie Z, Liu SJ, Bleris L, Benenson Y. Logic integration of mRNA signals by an RNAi-based molecular computer. Nucleic acids research. 2010; 38:2692-2701. [PubMed: 20194121]

51. Venkataraman S, Dirks RM, Ueda CT, Pierce NA. Selective cell death mediated by small conditional RNAs. Proceedings of the National Academy of Sciences of the United States of America. 2010; 71

52. Xie Z, Wroblewska L, Prochazka L, Weiss R, Benenson Y. Multi-Input RNAi-Based Logic Circuit for Identification of Specific Cancer Cells. Science. 2011; 333:1307-1311. [PubMed: 21885784]

53. Noireaux V, Bar-Ziv R, Libchaber A. Principles of cell-free genetic circuit assembly. Proceedings of the National Academy of Sciences of the United States of America. 2003; 100:12672-7. [PubMed: 14559971]

54. Shin J, Noireaux V. Efficient cell-free expression with the endogenous E. Coli RNA polymerase and sigma factor 70. Journal of biological engineering. 2010; 4

55••. Shin J, Noireaux V. An E. coli Cell-Free Expression Toolbox: Application to Synthetic Gene Circuits and Artificial Cells. ACS Synthetic Biology. 2012; 1:29-41.This work is the largest scale implementation to date of circuits constructed in complete transcription/translation systems using the endogenous $E$. coli transcriptional regulatory system.

56. Asahara H, Chong S. In vitro genetic reconstruction of bacterial transcription initiation by coupled synthesis and detection of RNA polymerase holoenzyme. Nucleic acids research. 2010; 38:e141. [PubMed: 20457746]

57. Karig DK, Iyer S, Simpson ML, Doktycz MJ. Expression optimization and synthetic gene networks in cell-free systems. Nucleic acids research. 2011 doi:10.1093/nar/gkr1191.

58. Ahn J-H, Kang T-J, Kim D-M. Tuning the expression level of recombinant proteins by modulating mRNA stability in a cell-free protein synthesis system. Biotechnology and bioengineering. 2008; 101:422-7. [PubMed: 18404770]

59. Ahn, J-ho; Keum, J-won; Kim, D-myung. Expression Screening of Fusion Partners from an E. coli Genome for Soluble Expression of Recombinant Proteins in a Cell-Free Protein Synthesis System. PLoS ONE. 2011; 6 
60. Ahn, J-ho; Keum, J-won; Kim, D-myung. High-Throughput, Combinatorial Engineering of Initial Codons for Tunable Expression of Recombinant Proteins research articles. Journal of Proteome Research. 2008; 7:2107-2113. [PubMed: 18386915]

61. Du L, Gao R, Forster AC. Engineering multigene expression in vitro and in vivo with small terminators for T7 RNA polymerase. Biotechnology and bioengineering. 2009; 104:1189-96. [PubMed: 19650080]

62. Karzbrun E, Shin J, Bar-Ziv R, Noireaux V. Coarse-Grained Dynamics of Protein Synthesis in a Cell-Free System. Physical Review Letters. 2011; 106:1-4.This quantitative work demonstrates advantages of cell-free protein synthesis by precisely modeling and experimentally verifying the rate of protein accumulation over time - essential knowledge for predictive design of proteinbased circuit elements.

63. Jewett MC, Forster AC. Update on designing and building minimal cells. Current opinion in biotechnology. 2010; 21:697-703. [PubMed: 20638265]

64••. Saito H, Kobayashi T, Hara T, Fujita Y, Hayashi K, Furushima R, Inoue T. Synthetic translational regulation by an L7Ae-kink-turn RNP switch. Nature chemical biology. 2010; 6:718.This work not only demonstrates tuning of an RNA-protein complex for logical integration but also successfully demonstrates in vitro design implementation in vivo.

65. Harris DC, Jewett MC. Cell-free biology: exploiting the interface between synthetic biology and synthetic chemistry. Current opinion in biotechnology. 2012 accepted.

66. Swartz JR. Transforming Biochemical Engineering with Cell-Free Biology. AIChE Journal. 2012; 58:5-13.

67. Carlson ED, Gan R, Hodgman CE, Jewett MC. Cell-free protein synthesis: Applications come of age. Biotechnology Advances. 2011 doi:10.1016/j.biotechadv.2011.09.016. 


\section{Highlights}

Simple in vitro biochemical circuits can provide insight into biological design.

Greater complexity of interactions in in vitro circuits decreases predictability.

Unanticipated applications derive from theoretically motivated work. 


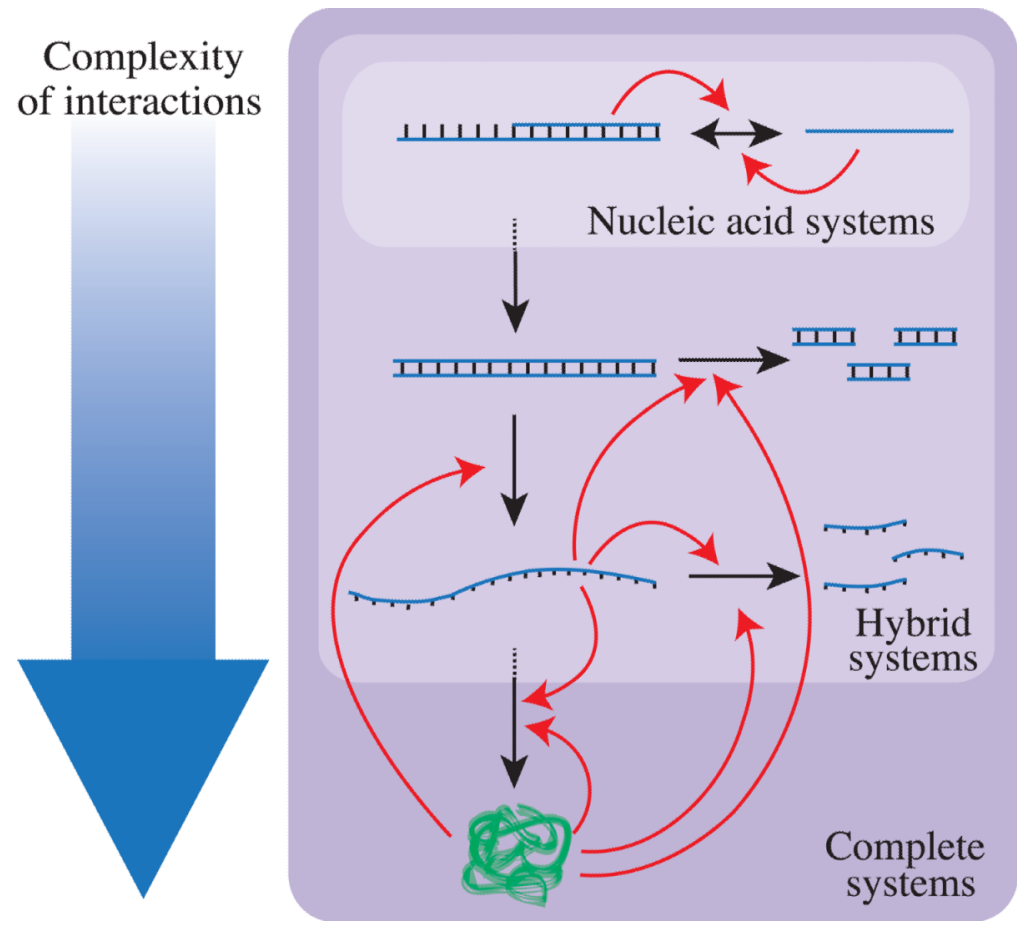

Figure 1.

The reactions and molecular composition of different synthetic in vitro circuits. Black arrows represent routes of information flow while red arrows represent interactions capable of controlling or modulating this flow. Nucleic acid based systems contain relatively few types of interactions, resulting in more predictable behavior. Hybrid systems, which are capable of producing and degrading nucleic acids, are intermediate on this scale with several new points of control and information flow. Complete systems can replicate the entire 'central dogma', but their increased complexity of interactions makes them less predictable. Note: small-molecule inputs and outputs via enzymes, ribozymes, and deoxyribozymes can, in principle, occur in all systems. 

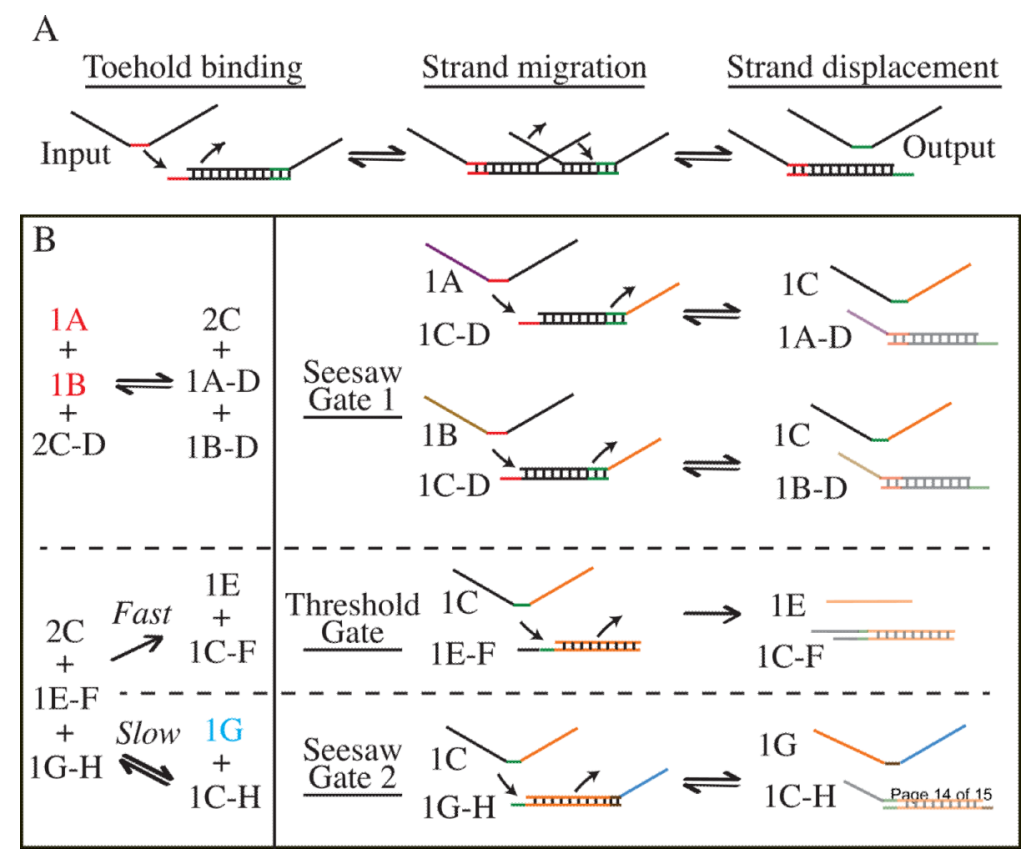

Figure 2.

Toehold exchange and seesaw gate function and implementation [13]. (A) A ssDNA input binds to a complementary toehold (red) on a seesaw gate and - through strand migration replaces an output strand of the dsDNA that is then capable of the reverse reaction via a separate toehold (green). (B) A functional AND gate that takes input molecules A and B and outputs $\mathrm{G}$. The left side presents a simplified overview while the right includes molecular detail. At seesaw gate 1, inputs (A and B) displace a strand of DNA (C) that then encounters threshold gate $1(\mathrm{E}-\mathrm{F})$. This gate quickly and irreversibly turns $\mathrm{C}$ into waste by having a larger complementary overhang than that of molecule G-H. Only after threshold molecules have been depleted will $\mathrm{C}$ bind to $\mathrm{G}-\mathrm{H}$ and produce the output strand $\mathrm{G}$. 


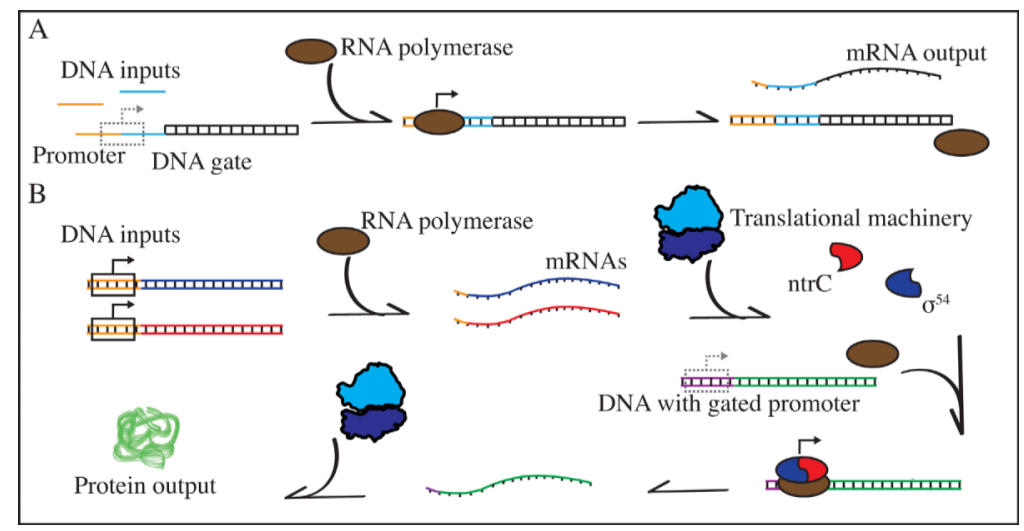

Figure 3.

Sample AND gate implementation in hybrid and complete systems. (A) Hybrid systems may use DNA, RNA, or small molecule inputs and outputs. Here, an incomplete promoter (dashed box) is bound by two ssDNA inputs that complete the promoter region. The polymerase enzyme (brown) then binds and transcribes an mRNA output. (B) Complete systems have a diverse array of possible inputs and outputs. Here, two DNA input signals with intact promoters (solid box) are transcribed into mRNA and translated into functional protein products ntrC (red) and $\sigma^{54}$ (blue). These protein products then bind to a polymerase and allow it to transcribe a gated promoter (dashed box), leading to production of a protein output [55]. 